\title{
Reinnervation of the biceps in C5-7 brachial plexus avulsion injuries: results after distal bypass surgery
}

\author{
Stefano Ferraresi, M.D., Debora Garozzo, M.D., and Paolo Buffatti, M.D. \\ Department of Neurosurgery, Ospedale S. Maria della Misericordia, Rovigo, Italy
}

\begin{abstract}
Object. The authors report various techniques, and their results, after performing median and ulnar nerve transfers to reanimate the biceps muscle in C5-7 avulsion-related brachial plexus injuries (BPIs).

Methods. Forty-three adult patients with BPIs of the upper-middle plexus underwent reinnervation of the biceps muscle; neurotization of the musculocutaneous nerve was performed using fascicles from the ulnar nerve (39 cases) and the median nerve (four cases). The different techniques included sectioning, rerouting, and direct suturing of the entire musculocutaneous nerve (35 cases); direct reinnervation of the motor branches of the musculocutaneous nerve (three cases); and reinnervation using small grafts to the motor fascicles that enter the biceps muscle (five cases).

Elbow flexion recovery ranged from M2 to M4+, according to the patient's age and the level of integrity of the hand. No surgery-related failure occurred. No significant difference in outcome was related to any of the technical variants. In patients younger than age 45 years and exhibiting a normal hand function a score of M4 or better was always achieved. On average, reinnervation occurred 6 months after surgery. There was no clinical evidence of donor nerve dysfunction.
\end{abstract}

Conclusions. When accurate selection criteria are met, the results after this type of neurotization have proved excellent.

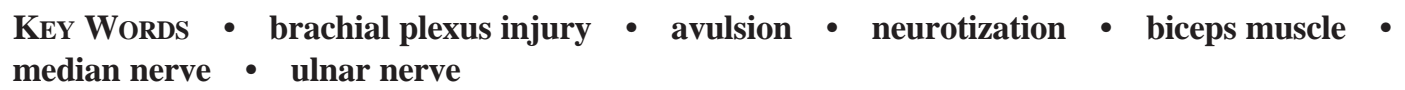

In C5-6 avulsion-related BPIs, the reinnervation of the biceps muscle is the primary goal. In March 1994, Oberlin, et al., ${ }^{7}$ described a pioneering technique for the neurotization of the motor branches of the musculocutaneous nerve. Using part of the ulnar nerve, they reported excellent results. The series included four patients in whom elbow flexion recovered earlier than 6 months after surgery and was scored M4.

Since 1995 we have performed surgery in 43 adult patients with an upper-middle BPI with avulsions at the upper nerve root level. Initially we strictly adhered to the original technique involving direct transfer of ulnar nerve fascicles onto the motor branches of the musculocutaneous nerve; later, however, we performed variations, preferring a direct suture between the donor site and the entire musculocutaneous nerve.

Moreover, in four cases, because of an anatomical variant ${ }^{3,8-10}$ (that is, a very low takeoff of the muscolocutaneous nerve, directly from the median nerve) a bypass from the median and the musculocutaneous nerves was attempted.

Abbreviation used in this paper: $\mathrm{BPI}=$ brachial plexus injury.

\section{CLINICAL MATERIAL AND METHODS}

Between 1995 and 2003, the procedure was performed in 43 consecutive adults in whom there was evidence of upper-region BPI and myelographic findings indicative of C-5 and C- 6 nerve root avulsions. All patients underwent surgery 2 to 12 months postinjury. The follow-up period ranged from 8 months (in the most recent cases) to 7 years.

Six patients had also suffered permanent impairment of wrist and finger extension due to an associated C-7 injury. All patients but four exhibited a normal function in the C-8 and T-1 innervated muscles. In these four cases intrinsic muscles and wrist flexors were weaker, although on the whole, their hand was functioning. Preliminary anatomical confirmation was obtained by examining the region in a cadaveric dissection: in the majority of cases, the biceps muscle is perforated by two major motor branches of variable length $(1-3 \mathrm{~cm})$ emerging from the musculocutaneous nerve. The donor nerve (ulnar in 39 cases, median in four) is exposed for a 5-cm-long tract in the upper third of the arm, and the epineurium is opened under microscopic visualization.

Electrical stimulation $(0.3-0.5 \mathrm{~mA})$ helps to identify a 
group of fascicles innervating the flexor carpi ulnaris (for the ulnar nerve) or the pronator teres flexor carpi radialis (for the median nerve). Afterward an internal neurolysis, carefully performed to avoid microvascular damage to the neighboring fascicles, focuses on the final donor area. Low-intensity electrical stimulations are repeated accordingly.

The cross-sectional area to be sacrificed never exceeds one fifth of the entire nerve. The fascicles are cut in a beveled fashion to allow the location of the recipient nerve (Video Clip 1).

Click here to view Video Clip 1. Detailed surgical technique for an ulnar-to-musculocutaneous bypass. The variant shown involves rerouting of the entire musculocutaneous nerve.

Recipient nerves included the motor branches of the musculocutaneous nerve in eight cases, neurotized with a direct suture in three patients or via small grafts $(2-3 \mathrm{~cm}$ long) in five cases. In 35 patients the entire musculocutaneous nerve was cut at its origin from the lateral cord, dissected, and distally rerouted to join the donor nerve. The two major fascicles of the musculocutaneous nerve were carefully freed from the connective tissue and sutured directly to the epineurium in the donor area. The goal is a perfect matching of the fascicles without intervening tissue; one to two 10-0 stitches and fibrin glue serve this purpose excellently (Fig. 1).

In four cases, the source of viable axons was the median nerve. This choice was considered obvious in the presence of an anatomical variant, namely a short musculocutaneous nerve leaving directly from the median nerve in the axilla (Fig. 2).

\section{RESULTS}

The overall results are summarized in Table 1. No failure has been observed. Excellent elbow flexion recovery (Grade M4 or M4+) was obtained in all groups if the patient was younger than 45 years of age and the wrist flexor, finger flexor, and intrinsic muscles functions were normal. Poor results (Grade M2) were observed in a 68-year-

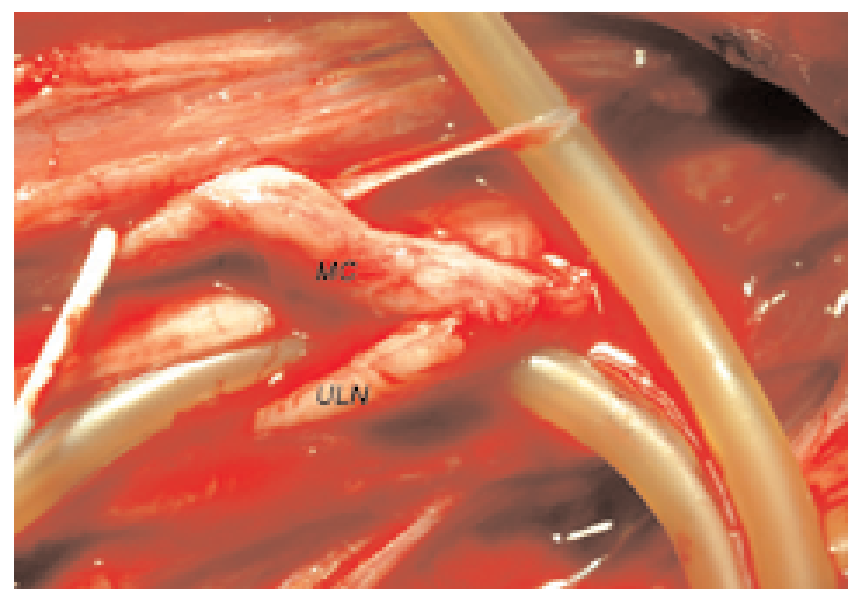

Fig. 1. Intraoperative photograph showing a rerouted musculocutaneous (MC) nerve sutured to the ulnar (ULN) nerve.

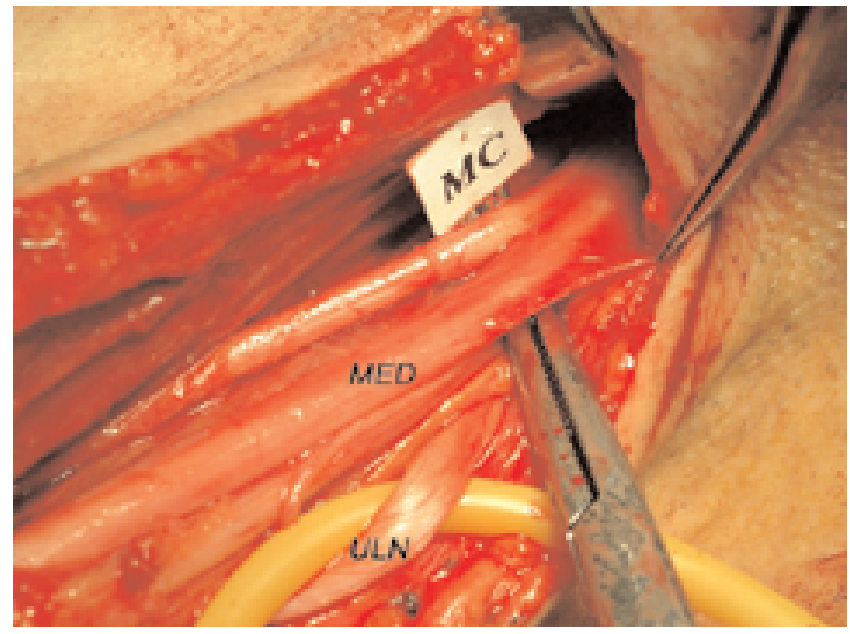

Fig. 2. Intraoperative photograph demonstrating the following anatomical variant: low origin of the musculocutaneous nerve from the entire median (MED) nerve below the axilla.

old patient and in those with weak flexor-intrinsic hand function.

The lack of wrist and finger extension, which occurred in C5-7 BPI avulsions, was not an obstacle; all patients in this group exhibited Grade M4/M4+ outcome. It also appears that success rate of the procedure was not affected by the technical variants. Direct suture or use of cable grafts, neurotization of selected motor branches, or rerouting and suturing of the entire musculocutaneous nerve were not associated with different results.

Postoperative time of recovery is usually short. Typically, function begins to return after 6 months and optimal strength after 9 to 10 months. A very early recovery (at 3 months) occurred, but this was not typical. In the patients with impairment of C8-T1 dependent muscles, signs of biceps muscle recovery never appeared earlier than 10 months.

There were very few donor site-related problems. In the early days, approximately $50 \%$ of the patients experienced a slight sensory disturbance (tingling in their fingers) but this rapidly disappeared. Strength was definitely not affected in patients with normal hand function or in those with impaired hand function. Long-term donor site morbidity was absent.

\section{DISCUSSION}

If candidates are properly selected (age $<45$ years and normal function of $\mathrm{C}-8$ and $\mathrm{T}-1$ dependent muscles), the ulnar/median-musculocutaneous nerve bypass procedure is a reliable technique to reinnervate the biceps muscle.

Although this finding is confirmed in the literature for the ulnar nerve transfer, ${ }^{1,4-7}$ there is no such evidence regarding the median nerve. ${ }^{4}$ This is probably because the median nerve is not an ideal candidate, at least in theory, being the lateral-most component directly involved in an upper-region BPI. Nevertheless, in our hands, both nerves were associated with an equal rate of biceps muscle reinnervation. This distal bypass technique is valuable because it provides a fast, precise, and powerful movement 
TABLE 1

Overall results in 43 patients who underwent neurotization procedures*

\begin{tabular}{|c|c|c|c|c|}
\hline \multirow[b]{2}{*}{ Procedure } & \multicolumn{4}{|c|}{ No. of Patients (function grade) } \\
\hline & $\begin{array}{l}\text { Normal Hand } \\
\text { Function } \\
\text { (age }<45 \text { yrs) }\end{array}$ & $\begin{array}{l}\text { Lack of Wrist \& } \\
\text { Finger Extension } \\
\text { (wrist flexor \& } \\
\text { intrinsics normal) }\end{array}$ & $\begin{array}{l}\text { Normal Hand Flexors } \\
\text { Function } \\
\text { (age }>60 \text { yrs })\end{array}$ & $\begin{array}{l}\text { Some Weakness in } \\
\text { Wrist or Intrinsics } \\
\text { (C-8 or T-2 } \\
\text { dural abnormality) }\end{array}$ \\
\hline $\begin{array}{l}\text { ulnar to entire } \\
\text { MC nerve }\end{array}$ & $20($ all w/ M4 or M4+) & 5 (all w/ M4) & $2(1 \mathrm{w} / \mathrm{M} 3 \& 1 \mathrm{w} / \mathrm{M} 2)$ & $4($ all w/ M2 or M2+) \\
\hline $\begin{array}{l}\text { median to entire } \\
\text { MC nerve }\end{array}$ & 3 (all w/ M4 or M4+) & $1(\mathrm{M} 4+)$ & & \\
\hline $\begin{array}{l}\text { ulnar to MC motor } \\
\text { branches }\end{array}$ & 3 (all w/ M4) & & & \\
\hline $\begin{array}{l}\text { ulnar to MC motor } \\
\text { branches via grafts }\end{array}$ & 5 (all w/ M4) & & & \\
\hline total $43 \mathrm{pts}$ & 31 & 6 & 2 & 4 \\
\hline
\end{tabular}

$* \mathrm{MC}=$ musculcutaneous.

at the elbow level in cases in which the upper roots are avulsed.

In the early recovery period, clenching of the fist is needed to activate the biceps muscle. Later, all the patients learn how to contract the biceps muscles with minimal movements of the wrist and little finger (Video Clip 2).

Click here to view Video Clip 2. Clinical result: clip shows the ability to contract the biceps muscle without clenching the fist. Also note the weight-bearing ability ( $2 \mathrm{~kg}$ for the bag and approximately $10 \mathrm{~kg}$ for the coat rack).

The absence of donor site morbidity and the possibility of a direct suture make this procedure also suitable for stretch injuries as an alternative to C5-6 graft repair.

The short period required for muscle recovery has now prompted us to undertake this procedure in elderly patients and those referred even 1 year after injury, regardless the type of C5-6 injury.

In the presence of a mixed-pattern injury of the upper plexus (for example, C-5 ruptured and C-6 avulsed), we prefer an ulnar to musculocutaneous bypass procedure to reanimate the biceps muscle and a graft repair from C-5

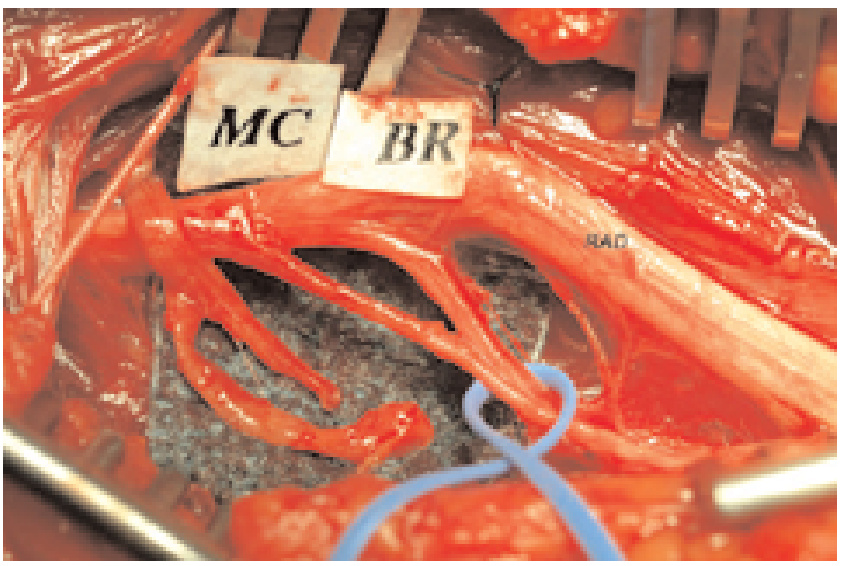

Fig. 3. Intraoperative photograph revealing two branches of the sensory musculocutaneous nerve (reinnervated via the ulnar nerve) to be sutured to the branches of the brachioradialis (BR) muscle. to reinnervate the shoulder muscles. In an analysis of results, we observed no significant differences among the various techniques in terms of outcome. We prefer to undertake the sectioning and rerouting of the entire musculocutaneous nerve because the procedure is straightforward and avoids the need for grafts. As a further application, in the most recent 10 cases, we also neurotized the sensory branch of the musculocutaneous nerve onto the nerve for the brachioradialis muscle (Fig. 3) in an attempt to retrieve some motor axons that would otherwise be dispersed.

In two cases (20\%), brachioradialis function was graded M2; however, the follow-up period has not been long enough to draw meaningful conclusions. A complete analysis of the technique-related results is beyond the scope of this paper.

In cases in which an avulsion injury of C-5 and C-6 nerves coexisted with a weakness of wrist and finger flexors (due to an additional partial injury of C-8 or T-1), biceps reinnervation is far less likely. Therefore, in these patients the aforementioned technique should not be applied if a validated alternative exists.

We prefer to undertake direct neurotization of the musculocutaneous nerve with the intercostal nerves and, more rarely, with the lower pectoral nerves. Although neurotization involving the pectoral nerves is commonly associated with good results, ${ }^{2}$ we rarely perform it (only in two of $>400$ brachial plexus procedures). Our reluctance is based on the need to preserve the integrity of the pectoralis muscle, which is useful for holding objects between the arm and the chest (thoracobrachialis pinch) in particular jobs, as well as because of its later use during secondary surgery. In fact, the pectoralis muscle (with mono- or bipolar transfer) may often be required to assist in elbow flexion. In cases in which the latissimus dorsi and teres major muscles are unavailable because they have been denervated, the latter is the only useful adjunct to improve extrarotation (with transfer of the distal tendon of the major pectoral muscle to the lateral side of the humerus).

\section{Disclaimer}

The authors received no financial support for this study. 


\section{S. Ferraresi, D. Garozzo, and P. Buffatti}

\section{References}

1. Bertelli JA, Ghizoni MF: Reconstruction of C5 and C6 brachial plexus avulsion injury by multiple nerve transfers: spinal accessory to suprascapular, ulnar fascicles to biceps branch, and triceps long or lateral head branch to axillary nerve. J Hand Surg Am 29:131-139, 2004

2. Blaauw G, Sloff AC: Transfer of pectoral nerves to the musculocutaneous nerve in obstetric upper brachial plexus palsy. Neurosurgery 53:338-342, 2003

3. Choi D, Rodriguez-Niedenfuhr M, Vasquez T, et al: Patterns of connections between the musculocutaneous and median nerves in the axilla and arm. Clin Anat 15:11-17, 2002

4. Hou Z, Xu Z: Nerve transfer for treatment of brachial plexus injury: comparison study between the transfer of partial median and ulnar nerves and that of phrenic and spinal accessory nerves. Chin J Traumatol 5:263-266, 2002

5. Loy S, Bhatia A, Asfazadourian H, et al: [Ulnar nerve fascicle transfer onto the biceps muscle nerve in C5-C6 or C5-C6-C7 avulsions of the brachial plexus. Eighteen cases.] Ann Chir Main Memb Super 16:275-284, 1997 (Fre)

6. Nascimento A, Teixeira RJ, Neri A, et al: Ulnar nerve fascicle transfer into the musculocutaneous nerve (biceps muscle) in C5-C6 brachial plexus injuries: 65 cases. J Hand Surg Am 28 (Suppl 1):7, 2003

7. Oberlin C, Beal D, Leechavengvongs S, et al: Nerve transfer to biceps muscle using a part of ulnar nerve for C5-C6 avulsion of the brachial plexus: anatomical study and report of four cases. J Hand Surg Am 19:232-237, 1994

8. Prasada Rao PV, Chaudhary SC: Communication of the musculocutaneous nerve with the median nerve. East Afr Med J 77:498-503, 2000

9. Saeed M, Rufai AA: Median and musculocutaneous nerves: variant formation and distribution. Clin Anat 16:453-457, 2003

10. Uysal II, Seker M, Karabulut AK, et al: Brachial plexus variations in human fetuses. Neurosurgery 53:676-684, 2003

Manuscript received March 13, 2004.

Accepted in final form April 2, 2004.

Address reprint requests to: Stefano Ferraresi, M.D., Department of Neurosurgery, Ospedale S. Maria della Misericordia, Via Tre Martiri, 89, 45100 Rovigo, Italy. email: nchro@libero.it. 